\title{
Ischemic stroke following Dengue fever: A case report
}

\author{
Robin George Manappallil \\ Physician, Department of Medicine, National Hospital, Calicut, Kerala, India
}

Dengue fever is an acute febrile illness associated with severe headache, myalgias, arthralgias, rashes, leucopenia and thrombocytopenia. Occasionally unusual haemorrhage such as gastrointestinal bleeding, intracranial haemorrhage, hypermenorrhoea, hematuria and massive epistaxis occur. This is a case of an elderly male with dengue fever who presented with ischemic stroke.

Key words: Dengue fever, Ischemic stroke, vasculitis
Access this article online Website:

http://nepjol.info/index.php/AJMS

DOI: 10.3126/ajms.v7i2.13340

E-ISSN: 2091-0576

P-ISSN: 2467-9100

\section{INTRODUCTION}

Dengue fever (DF) is the fastest emerging arboviral infection spread by Aedes mosquitoes with major public health consequences in over 100 tropical and sub-tropical countries in South East Asia, the Western Pacific and South and Central America. It is an acute febrile illness associated with severe headache, myalgias, arthralgias and rashes. Blood investigations show leucopenia and thrombocytopenia. Neurological manifestations in the form of stroke are uncommon but patients presenting with intracranial bleed during the course of DF have been reported. ${ }^{1}$

This case report highlights the possibility of developing ischemic stroke in a patient suffering from dengue fever with thrombocytopenia.

\section{CASE HISTORY}

The patient being reported in this case is an 86 year old male, who presented with acute onset weakness of right half of the body, with deviation of angle of mouth to the left and slurred speech, of 1 day duration. He is not a diabetic or hypertensive. He did not have any history of ischemic or valvular heart disease. He was having high grade fever for the past 1 week.

On examination, he was conscious and oriented. He had a temperature of $101{ }^{\circ} \mathrm{F}$. His pulse rate was $60 /$ minute which was regular, blood pressure of 160/100 $\mathrm{mmHg}$ and respiratory rate of 22 breaths/minute. Neurological examination revealed right upper motor neuron facial palsy with right sided hemiplegia, with brisk deep tendon reflexes and an extensor plantar reflex on the right side. There was no sign of meningeal irritation. Other systemic examinations were normal. His computed tomography brain showed multiple lacunar infarcts as shown in Figure 1. Blood investigations revealed mild leucocytosis with thrombocytopenia (71,000/microliter) and mildly elevated liver enzymes. His Dengue NS1 antigen was positive. His malarial smear, leptospirosis serology, scrub serology and blood cultures were negative. His ECG, echocardiogram and carotid artery doppler were normal. Antinuclear antibodies, homocysteine, protein $\mathrm{C}$ and $\mathrm{S}$ levels were normal. Cerebrospinal fluid analysis was normal as shown in Figure 1.

Patient was treated conservatively with limb physiotherapy. His serial platelet counts decreased over the next 2 days but later showed a rising pattern, reaching up to 86,000/microliter. He also became afebrile. However, on day 4 of his hospital stay, patient had an episode of vomiting followed by aspiration pneumonia. He was intubated, put on mechanical ventilation and started on intravenous antibiotics. But patient went into severe sepsis and shock and expired on day 7 of hospital admission.

\section{DISCUSSION}

According to the WHO, dengue is the most rapidly spreading mosquito borne viral disease in the world. There 


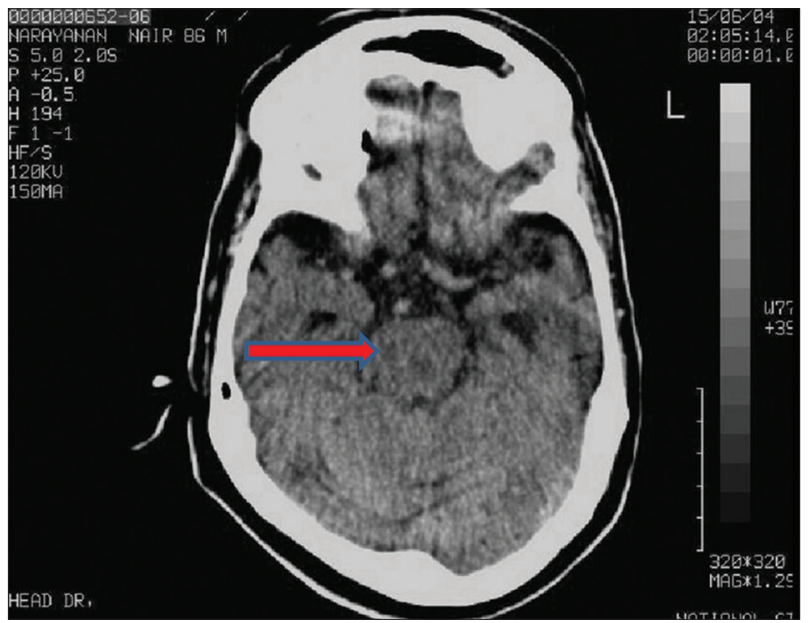

Figure 1: Acute pontine infarcts

has been a 30 fold increase in the incidence rates over the past 50 years. The infection is transmitted from human to human by the bite of Aedes mosquitoes. Humans and monkeys serve as reservoirs. The viraemia among humans builds up 2 days before the onset of the fever and lasts for 5-7 days after the onset of the fever. During these two periods the vector species gets infected and thereafter, the humans become dead-ends for transmission. ${ }^{2}$

The virus has 4 serotypes (DENV 1, 2, 3, 4). Infection with any one serotype confers lifelong immunity to that serotype. Although all four serotypes are antigenically similar, they are different enough to elicit cross-protection for only a few months after infection by any one of them. ${ }^{2}$

Dengue virus infection may be asymptomic or can present as dengue hemorrhagic fever with or without shock. There may also be organ involvement. ${ }^{2}$ The incubation period is 4-10 days. DF is defined as an acute febrile illness of
2-7 days duration with two or more of the following i.e. headache, retro-orbital pain, myalgia/arthralgia, rash, haemorrhagic manifestation (petechiae, positive tourniquet test) and leucopenia. Dengue Hemorrhagic Fever will have addition of bleeding manifestations, thrombocytopenia and signs of plasma leakage. The disease is diagnosed by virus isolation, nucleic acid detection (PCR) and antigen detection (NS1) during the early phase while serology is done at the end of the acute phase. ${ }^{2}$

Encephalitis, myelitis, Guillain-Barré syndrome and myositis are the neurological complications of dengue infection. ${ }^{3}$ Intracranial haemorrhage due to thrombocytopenia have been reported, but ischemic stroke is a rare complication of dengue infection. ${ }^{4,5}$ Meningovasculitis has been regarded as a probable mechanism for the occurrence of ischemic stroke in DF. Another possibility is the development of a transient hypercoagulable state during dengue infection. In this case, the probable cause for ischemic stroke could have been vasculitis.

\section{REFERENCES}

1. Kumar R, Prakash $\mathrm{O}$ and Sharma BS. Intracranial hemorrhage in dengue fever: management and outcome: a series of 5 cases and review of literature. Surg Neurol 2009; 72(4):429-433.

2. WHO. Dengue and dengue haemorrhagic fever. Factsheet $N^{\circ} 117$, revised March 2009. Geneva, World Health Organization, 2008.

3. Verma R, Sharma P, Garg RK, Atam V, Singh MK and Mehrotra HS. Neurological complications of dengue fever: Experience from a tertiary center of north India. Ann Indian Acad Neurol 2011;14:272-278.

4. Liou LM, Lan SH and Lai CL. Dengue fever with ischemic stroke: A case report. Neurologist 2008;14:40-42.

5. Mathew $S$ and Pandian JD. Stroke in patients with dengue. J Stroke Cerebrovasc Dis 2010; 19:253-256. 